\title{
First REP-LECOTOX Workshop-ecotoxicogenomics: the challenge of integrating genomics/proteomics/metabolomics into aquatic and terrestrial ecotoxicology
}

\author{
R. Kovacevic • I. Teodorovic • S. Kaisarevic • \\ I. Planojevic $\cdot$ S. Fa • V. Dakic $\cdot$ K. Pogrmic $\cdot$ S. Virijevic
}

Received: 3 December 2008 / Accepted: 14 December 2008/Published online: 21 March 2009

(C) Springer-Verlag 2009

The Workshop "Ecotoxicogenomics: The challenge of integrating genomics/proteomics/metabolomics into aquatic and terrestrial ecotoxicology" held in Novi Sad, Serbia, at UNSFS, Department of Biology and Ecology, organized as a part of the Project INCO-CT-2006-043559-REP-LECOTOX, brought together experts in the field of genomics, transcriptomics, proteomics, metabolomics, and bioinformatics. Five plenary lectures and seven lectures on the specific themes, as well as discussion afterwards, gave to participants a comprehensive insight into the possibility of application of omic technologies in the field of ecotoxicology. The workshop was attended by approximately 50 participants mostly from Romania, Bulgaria, Croatia, Macedonia, Montenegro, Bosnia and Herzegovina, and Serbia (Fig. 1).

The Workshop program was prepared by the Scientific Committee: J.K. Chipman, S. Scholz, K. Hilscherova, and R. Kovacevic.

In the first keynote lecture "Overview of ecotoxicogenomics," Prof. J.K. Chipman (School of Biosciences, University of Birmingham, UK) stressed that a major advantage of functional genomics technologies, which enable measurements of thousands of transcripts, proteins, and metabolites, is their "open" nature that does not require prior assumptions about the choice of biomarkers, thus, being particularly valuable to assess mechanisms of action

Responsible editor: Walter Giger

ESPR Special Issue-ESTROM

R. Kovacevic $(\bowtie) \cdot$ I. Teodorovic $\cdot$ S. Kaisarevic $\cdot$ I. Planojevic $\cdot$ $\mathrm{S} . \mathrm{Fa} \cdot \mathrm{V}$. Dakic $\cdot$ K. Pogrmic $\cdot$ S. Virijevic

Department of Biology and Ecology, Faculty of Sciences,

University of Novi Sad,

D. Obradovica Sq. 2,

21000 Novi Sad, Serbia

e-mail: radmilak@ib.ns.ac.yu and the effect of mixtures of chemicals where unknown biological targets may be involved. He also pointed out that attention needs to be given to distinguishing between compensatory, adaptive, and toxic responses and to discovering patterns of change that are diagnostic and predictive. Many interesting examples of "omic" responses were shown together with discussion and proposals for use of such data in ecological monitoring and risk assessment.

In the second keynote lecture "Environmental transcriptomics-predicting adverse and long-term effects," Dr. Stefan Scholz (Department for Cell Toxicology, UFZ, Leipzig, Germany) showed that information obtained by whole genome analysis can be useful if they are linked to appropriate applications, such as the identification of mechanisms of action or biomarkers for biomonitoring and the prediction of (long-term) adverse effects. His presentation highlighted two examples demonstrating applications in ecotoxicology and/or regulatory testing: the first one was the application of gene expression as a marker for long-term adverse effects of endocrine disrupting chemicals, while the second highlighted example described the potential application of gene expression markers for the prediction of chronic fish toxicity by extending the fish embryo test from acute to chronic toxicity test.

In the third keynote lecture "Environmental proteomics," Dr. Thomas Knigge (Laboratoire d'EcotoxicologieMilieux Aquatiques, Université du Havre, France) pointed out that the proteomic profile represents a link between effects at the molecular and whole organism level, since proteins are the first functional level directly or indirectly affected by toxicants. Showing the results of several different studies using gel and non-gel-based proteomics, he illustrated some of the practical and conceptual difficulties when applying proteomics to ecotoxicological studies. He stressed the needs for sophisticated bioinformatics and 


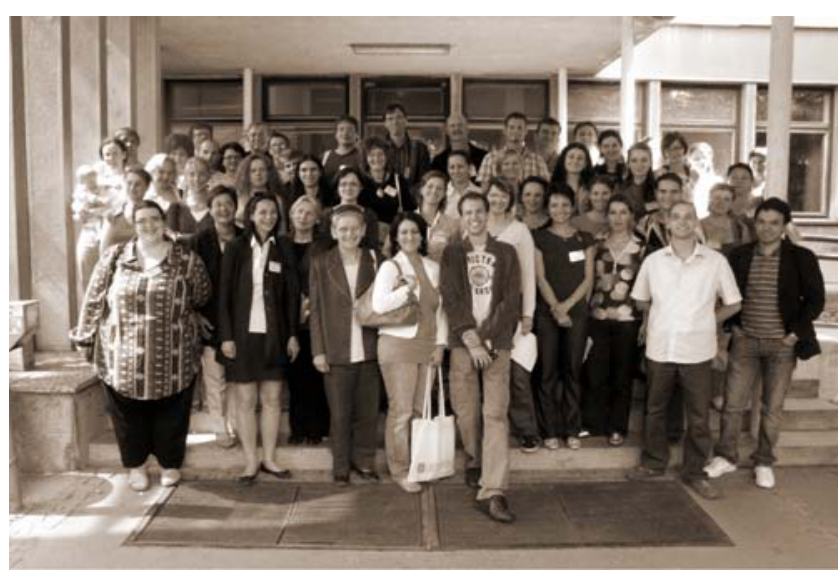

Fig. 1 Workshop participants

for quality control procedures which might increase the reproducibility.

Dr. Jake Bundy (Biomolecular Medicine, Sir Alexander Fleming Building, Imperial College, UK) in his keynote lecture "Environmental metabolomics" stressed the important advantages and drawbacks of metabolomics. Apparently, the biggest advantage of metabolomics is that it represents the most direct measure of overall cellular functional state - an alternative measure of phenotype. Metabolomics facilitate studies with genetically uncharacterized organisms, as metabolites are universal, unlike genes or proteins. Among the advantages, he mentioned high precision and high throughput. However, he also listed many drawbacks like the appearance of unusual secondary metabolites which are currently difficult to assign. He also stressed that the results obtained depend critically on the analytical method chosen and presented the main platforms for metabolite profiling: proton nuclear magnetic resonance spectroscopy and mass spectrometry.

The fifth introductory lecture was on Environmental Bioinformatics: Renzo Kottmann (PhD student, Microbial Genomics Group, Department of Molecular Ecology, Max Planck Institute for Marine Microbiology, Bremen, Germany) presented results on Bioinformatics for Marine Ecological Genomics.

Lectures on specific themes "Biomarkers of aquatic toxicology: past, present, and future" presented by Dr. Ron van der Oost (Waternet, Amsterdam, The Netherlands) and "Reporter gene assays and specific gene expression in biomonitoring of the contaminated environmental matrices" by Dr. Klara Hilscherova (RECETOX, Brno, Czech Republic) stressed the importance of application of a battery of sensitive and simple bioassays, especially in vitro assays in assessment of complex samples.
Lectures: "Introduction of toxicoproteomic approach with Tetrahymena thermophila in environmental monitoring," Prof. Romana Marinšek-Logar (University of Ljubljana, Slovenia), "Bacterial community structure analysis in terrestrial and aquatic environments - an ecotoxicogenomic approach," Dr. Rok Kostanjšek (University of Ljubljana, Slovenia), and "Immunotoxicity of xenobiotics as an aspect of their ecotoxicity," Prof. Milena Kataranovski (Institute for Biological Research "Siniša Stanković", Belgrade, Serbia) presented their research on different model systems, while the last lecture "State of ecotoxicology research and legislation in Serbia" by Dr. Ivana Teodorovic (LECOTOX, UNSFS, Novi Sad, Serbia) gave the overview of the recent and current ecotoxicological research in Serbia.

The workshop has highlighted a range of molecular techniques which could complement whole organism testing and contribute to ecotoxicological risk assessment, promoting the benefits of a multidisciplinary approach. It has been concluded that omics show high potential to become an integral part of ecological risk assessment strategies and that toxicity testing of the twenty-first century would certainly rely on omic technologies.

After the lectures, laboratory sessions were organized: Workshop session "7900HT Fast Real-Time PCR System with 7900HT TaqMan Low Density Array Block demonstration of work with Micro Fluidic Card held by the representative of Applied Biosystems" and Workshop session "Agilent microarray for toxicogenomics research" and "Lab-on-a-ChIP technology" led by the Agilent representatives with live demonstrations.

The Workshop successfully fulfilled many of the foreseen objectives. Apart from giving the comprehensive overview of application of genomics-based tools in ecotoxicology, it contributed to popularization of ecotoxicogenomic and ecotoxicological research in general in Serbia as well as in the region which, due to specific historical problems and current socioeconomic conditions, can be recognized as the region with the most enhanced problems of environmental pollution, including hazards from numerous hot spots of severe terrestrial and aquatic contamination. Above all, the workshop offered the unique opportunity for networking between some of the best European institutions and researchers and professionals, including the end users in the field of environmental protection from the Western Balkan Region. The workshop surely represents a milestone in contemporary approach to ecotoxicological research in the region.

All workshop presentations in pdf form, as well as other information about REP-LECOTOX project are available at the official LECOTOX web site www.lecotox.net. 